also known as dyspnea. The exact determining of etiology is still a main diagnostic challenge.

Standard methods include: taking a history, clinical examination, auscultation and radiological procedures. But for many reasons, such as exposure to ionizing radiation and the inability to apply at bedside the chest radiography is not any more 'Gold standard' to acute respiratory insufficiency.

Lung ultrasound (LUS) has been shown to have great sensitivity and specificity in the differential diagnosis of the most common respiratory conditions. This method can be applied bedside, it can be used even outside of hospitals, follow - up is easy and does not expose the patient to harmful ionizing radiation.

According to a diagnostic algorithm called 'BLUE protocol' established by Daniel Lichtenstein lung ultrasound has been proven to be accurate in finding the true cause of respiratory insufficiency in a large number of cases.

Major acute respiratory disorders are pulmonary edema, pulmonary inflammation, acute respiratory distress syndrome (ARDS), pulmonary embolism, asthma exacerbation and chronic obstructive pulmonary disease (COPD), pleural effusion and pneumothorax.

Aim To demonstrate the role of lung ultrasound (LUS) as the first line of pulmonary condition diagnosis, to define the role of LUS during folow-up visits in order to prevent complications, to increase awareness of LUS importance in relation to the most frequent pulmonary disease in pediatric patients.

Materials-methods We have described the LUS examination technique as normal LUS images and LUS findings in the most frequent pulmonary pediatric diseases.

Results and conclusion LUS has been proven to be a very important diagnostic tool in almost all lung disease in pediatric disease described but obviously still need continuous research to explore its potential.

\section{P557 REVIEW OF INCIDENCE AND INDICATION OF MICTURATING CYSTOURETHROGRAM (MCUG) IN PAEDIATRIC PATIENTS IN CORK UNIVERSITY HOSPITAL (CUH)}

${ }^{1}$ Carol Stephens*, ${ }^{2}$ Sean Flanagan. 'Paediatric Department, Cork University Hospital, Cork, Ireland; ${ }^{2}$ Radiology Department, Cork University Hospital, Cork, Ireland

\subsection{6/archdischild-2019-epa.891}

Micturating Cystourethrogram (MCUG) is commonly uses to investigate Vesocoureteroreflux (VUR). While the test lends itself to a high sensitivity and specificity rate in identification of VUR it doesn't come without risks. The biggest challenge is practicality and tolerability. MCUGs are not pleasant and the window of opportunity to perform them without sedation is narrow. Furthermore, MCUG is an invasive test predisposing the patient to iatrogenic infection. Keeping the above in mind, it is essential that MCUGs are only performed in those who need them and will influence their future management and treatment

\section{Aims}

1. Identify all children in who had a MCUG at less than two years of age

2. Identify the indication for MCUG

3. Investigate if reason for MCUG met guidelines.
Methods A retrospective review of MCUGs performed in CUH in all children less than two years of ages between 01/ 01/15-31/12/17.Patients from Cork University Maternity Hospital were included.Patients were identified via IMPAX Radiology system. Nice Guidelines were used as the standard.

Results

Ninety nine children were identified

\begin{tabular}{lc}
\hline Appropriate Indications & 95 \\
\hline Inappropriate & 4 \\
\hline Indications & \\
\hline
\end{tabular}

\begin{tabular}{ll}
\hline INDICATION & NUMBER \\
\hline Atypical UTI & 20 \\
Recurrent UTI & 1 \\
Hydronephrosis & 68 \\
Scarring & 1 \\
Other & 5 \\
\hline
\end{tabular}

- Two children met more than one indication; atypical UTIs and hydronephrosis

- One child had more than three indications; hydronephrosis, scarring and 'other'

The main reasonfor not meeting criteria was for recurrent UTIs outside the $<6$ month of age period.

Discussion MCUG is recommended in children who have had a renal ultrasound revealing hydronephrosis, scarring or other findings that may suggest high grade reflux or in children less than six months with atypical and or recurrent UTIs. There is considerable controversy regarding the optimal management of VUR varying between prophylactic antibiotics versus surgery. Early identification optimizes kidney preservation. By defining risk, early stratification allows earlier identification of high risk children .Incidence of VUR has been difficult to ascertain but it has been estimated that $0.8-1.4 \%$ of normal children will have reflux. This incidence soars to $30-50 \%$ in children with UTIs depending on age and a higher incidence in males despite a higher incidence of UTIs in females.

In our hospital, we adhere to current best medical practice and don't perform MCUGs when not indicated. This is important given the significant risks associated with MCUGs.

Conclusion The majority of MCUGs were performed in line with current guidelines. The most common indication was hydronephrosis. There is good knowledge among paediatric and radiology teams of the best practice.

\section{P558 A RETROSPECTIVE STUDY OF MANAGEMENT OF HENOCH SCHONLEIN (HSP) NEPHRITIS IN CHILDREN IN IRELAND A}

Carol Stephens*, Tracy Conlon, Mary Waldron, Maria Stack. OLCHC, Dublin, Ireland

10.1136/archdischild-2019-epa.892

HSP is a prevalent disease with an incidence of 6-24 per 100,000.(1) It can lead to significant chronic disease with 
renal involvement being the principal cause. It requires close monitoring and treatment. Renal biopsies can be important prognostic markers and guide best management.(2) Currently, there have been no significant randomised controlled trials detailing best management and hence treatment varies significantly. This study reviews practice in a national tertiary renal unit in Ireland over a fifteen-year period.

Aims 1. Identify children who had a renal biopsy.

2. Review Medical management, duration of therapy and follow up.

Methods This was a retrospective review between September 2001 to April 2017. Data was collated by computer and paper records, online haematological and radiological resources. This review excludes children managed in another tertiary centre in Ireland.

Results

The main outcomes were patient demographics, (i) Biopsy findings: Acute vs Chronic and staging classification, (ii) Drug Treatment and Duration (iii) Follow up: urinanalysis at interval time points, renal ultrasonography and renal function. Thirty children had a renal biopsy. 10 For persistent proteinuria, 2 for recurrent episodes, deranged renal function, mixed nephritic/nephrotic, IgA vs HSP respectively and 12 not classified.

$73 \%$ required medical treatment, the majority polypharmacy. When immunosuppressants were needed a combination of steroids and Mycophenolate Acid were most commonly used. $73 \%$ required medical treatment for over eighteen months with evidence of proteinuria often guiding duration.

Regular follow up included urinanalyis, renal bloods, ultrasonography and blood pressure measurement.

To standarise care nationally, a basic guideline was created to aid appropriate referral to our tertiary centre.

Conclusion HSP is rare but can cause significant morbidity. RCTs are required to determine best management and to standardize care nationally and internationally

\section{P559 A POSSIBLE PATHOGENIC RELATIONSHIP BETWEEN ENURESIS AND INNOCENT HEART MURMURS}

${ }^{1}$ Pietro Ferrara*, ${ }^{2}$ Federica Di Ruscio, ${ }^{2}$ Margherita Zona, ${ }^{2}$ Roberta Autuori, ${ }^{2}$ Alessandro Di Lucia, ${ }^{2}$ Flavia Dosa, ${ }^{2}$ Giulia Franceschini, ${ }^{2}$ Diletta Saitta, ${ }^{2}$ Michela Pulcino, ${ }^{1}$ Ester Del Vescovo, ${ }^{3}$ Massimo Pettoello-Mantovani. ${ }^{1}$ Institute of Pediatrics, Università Cattolica del Sacro Cuore, Rome, Italy; ${ }^{2}$ Campus Bio-Medico University, Rome, Italy; ${ }^{3}$ Department of Pediatrics, Scientific Institute Casa Sollievo della Sofferenza, University of Foggia, Foggia, Italy

\subsection{6/archdischild-2019-epa.893}

Objective The aim of this study is to evaluate the prevalence of innocent heart murmurs in children affected by nocturnal enuresis (NE). Specifically, for this purpose, a possible pathogenic relationship linking both conditions.

Design This study is an observational study.

Setting The study was characterized by a careful examination of patient's medical history and performing of physical examination, including the weight and height measurement. Mostly, it was a cardiological examination including monitoring patient's blood pressure and abnormal heart sounds: patent's foramen ovale, slight systolic impurity, mitral insufficiency, muffled heart sound, heart murmur, and impurity. The study was carried out in compliance with the Helsinki Declaration. Analyses were conducted using the Microsoft Excel 2011 (version 14.0.0) program. We used descriptive statistics to describe patients' information (age, gender, familiarity). Data were expressed as the mean \pm standard deviation and percentage. The $\chi^{2}$ test was used for categorical variables. The significance level was set at $p<0.05$.

Patients We are enrolling about 400 children (G1), 300 males and 100 females aged between 5 and 15 years, affected from NE referred to the Service of Pediatrics, 'Campus Bio-Medico' University Hospital of Rome, from September 2013 to January 2019 in the study. The control group is composed of about 400 children without NE (G2).

Main outcome measures Prevalence of innocent heart murmurs in children with MNE or N-MNE.

There isn't any study investigating the association of NE with innocent murmur in the literature.

Results The prevalence of heart innocent cardiac murmur appears to be significantly higher in children who suffer from NE. The results obtained are not definitive, because the study is ongoing.

Conclusions These findings made us acquaintance of the presence of possible underlying mechanisms which explain the association between higher prevalence of innocent heart murmur and enuresis, further studies are needed. A possible explanation of this relationship could be provided by heart increased secretion of atrial natriuretic peptide (ANP) and Btype natriuretic peptide (B-BNP). In particular, the murmur could be accentuated by hypervolemia, which in turn determines atrial and ventricular distention. This stimulates cardiomyocytes into producing ANP and B-BNP. ANP shares with BBNP a high degree of structural homology and a profile of diuretic, natriuretic and vasodilator activities and inhibition of the renin-angiotensin-aldosterone system.

\section{P560 URINARY SCREENING IN 6-12 YEARS SCHOOL CHILDREN IN ' MAKKAH \& AL-BAHA REGIONS' IN SAUDI ARABIA}

Mahmoud Rashad*. King Fahd Hospital, Al Baha, Saudi Arabia. Al Azher University, Cairo, Egypt

10.1136/archdischild-2019-epa.894

Introduction and aim of the work

Mass urinary screening is a useful tool to identify children with asymptomatic progressive renal diseases. Patients with renal disease have a variety of different clinical presentations, that are directly referred to the kidney or to extra renal clinical manifestation. Proteinuria as well as hematuria may be the only early signs of renal disease. UTI is very common in children with severe consequences on the kidney function leading to chronic kidney disease (CKD) and hypertension if left untreated. Hypercalciuria is the most common cause of urolithiasis in children.

The purpose of this study is to screen elementary school children in Makkah and Al-Baha region in Saudi Arabia, for prevalence of hematuria, proteinuria and hypercalciuria. The goal for screening of healthy individual or population for diseases is to reduce morbidity and mortality through early detection and treatment.

Subjects and methods This cross sectional study, approved by the Institutional Review Board Committees of Makkah and Al Baha, and was carried out between October 2016 and April 2017 on randomly selected 12352 children (including 9299 children from Makkah and 5053 children from Al Baha). Dipstick test was performed by a pediatrician. Children with abnormal urinary findings in the second screening were 